\title{
Fratura radicular horizontal: manejo conservador
}

\author{
Horizontal root fracture: conservative management
}

COSTA, Isabella Eduarda Bozutti da'; MARTINS, Milena Perraro²; DUQUE, Jussaro Alves²; BOER, Nilton César Pezati ${ }^{3}$; DUARTE, Marco Antonio Hungaro ${ }^{4}$; FERNANDES, Samuel Lucas ${ }^{5}$

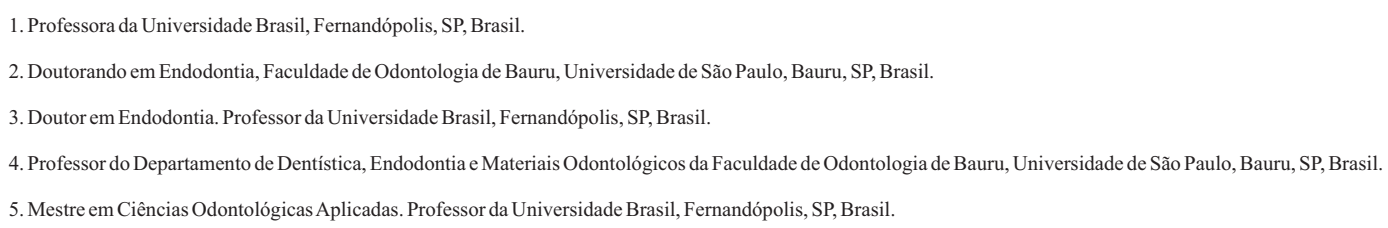

Recebido: 26.10 .2017

Aceito: 12.11.2017

\section{RESUMO}

A manutenção do dente em função e com uma estética favorável na cavidade bucal consiste em um dos principais objetivos da odontologia. Os traumatismos alvéolo-dentário por vezes se apresenta como um desafio na clínica odontológica, principalmente no caso de fratura radicular. O objetivo deste artigo é exibir um caso clínico onde o paciente que sofreu um traumatismo levando a uma fratura horizontal, e foi adotada uma conduta conservadora.

Palavras-chave: Traumatismos dentários. Tratamento conservador. Cavidade pulpar.

\author{
ABSTRACT \\ Tooth in functional and favorable aesthetics in oral cavity is one of the main objective of dentistry. Alveolus-tooth trauma is \\ sometimes a challenge in the dental clinic, especially in the case of root fracture. The purpose of this article is to show one a \\ case where the patient who stricken trauma and horizontal root fracture. Conservative approach was adopt.
}

Keywords: Tooth injuries. Conservative treatment. Dental pulp cavity. 


\section{INTRODUÇÃO}

O trauma dentoalveolar é uma lesão traumática que pode envolver basicamente estruturas como alvéolo, dentes e tecidos moles ${ }^{1}$. Em dentes pode variar de uma simples fratura de esmalte ou pode levar a perda do elemento dentário. O trauma pode ser consequência de acidentes esportivos ou automobilísticos, queda, maus tratos, lutas, brigas ${ }^{2-3}$ e na maioria das vezes acomete crianças no período escolar e adolescentes $^{1,4}$. Essas situações de urgência são experiências dramáticas para os pais e para as crianças, uma vez que envolvem traumatismos em região de cabeça e pescoço ${ }^{5}$.

Os traumatismos podem ser classificados em: fratura de esmalte; fratura de esmalte e dentina; fratura coronária; fratura de coroa e raiz; fratura radicular e fratura da parede e processo alveolar. As fraturas radiculares horizontais alteram prontamente a estrutura dentária rompendo a raiz e envolvendo dentina, polpa e cemento ${ }^{6}$. Essas fraturas podem acometer o terço cervical, médio ou apical, embora seja mais prevalente no terço médio7. Esses dentes frequentemente apresentam mobilidade, extrusão e deslocamento do fragmento coronal em diferentes graus, dependendo da localização da fratura e da gravidade do traumatismo .

O diagnóstico pode ser dado por características clínicas como mobilidade dental, deslocamento do fragmento e sensibilidade a palpação na raiz, mas a sua confirmação se dará por exames de imagem?. O prognóstico dos traumas radiculares é dependente do local fraturado, que pode ser no terço cervical médio ou apical da raiz, do grau de deslocamento dos fragmentos, da situação da polpa, da oclusão e do estado oral geral do paciente ${ }^{10-12}$. O objetivo deste relato é apresentar um caso clínico onde o paciente sofreu um traumatismo levando a uma fratura horizontal, e foi adotada uma abordagem conservadora.

\section{RELATO DE CASO}

O paciente do sexo masculino de 12 anos de idade apresentou-se a clínica após um traumatismo em um jogo de futebol. A queixa principal apresentada foi: "o dente ficou torto e dói muito". Ao exame físico observou-se ferimentos nos lábios e mucosa compatíveis com a descrição do acidente durante o jogo de futebol. Observouse ainda que o elemento 11 apresentava-se fora de posição e então realizou-se o exame radiográfico periapical, constatando uma fratura horizontal.

Após conversar com o responsável sobre as opções de tratamento e explicação da viabilidade da manutenção do dente, com o reposicionamento e Splint, o aceite pelo tratamento foi de pronto, tanto por ele quanto pelo paciente. A conduta clínica foi o reposicionar do dente com auxílio de um fórceps 150, após anestesia infiltrativa com solução estéril injetável de mepicavaina $2 \%$ com epinefrina 1:100.000. Foi realizada um Splint rígido e o dente foi mantido sem a realização do tratamento endodôntico e o paciente recebeu acompanhamento semanal no primeiro mês, e mensal durante seis meses.

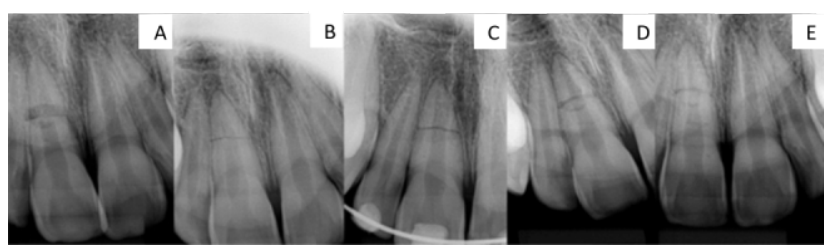

Figura 1 - Sequência evolutiva do caso. Sendo a radiografia inicial (A); após o reposicionamento (B); após o Splint (C); após a remoção da explintagem (D); controle de dois anos (E).

Após um mês o Splint foi removido e o dente manteve a sensibilidade pulpar, sendo assim optou-se por mantê-lo sem intervenção endodôntica. Após o controle de dois anos o dente manteve-se vital e sem alteração de cor.

\section{DISCUSSÃO}

O paciente apresenta características comuns entre os atendidos com traumatismos alvéolo-dentários. Que são pacientes jovens ${ }^{5,8,13}$ e majoritariamente do sexo masculino $0^{3,5,8,13}$. A etiologia relacionada ao caso é comum, umas vez que os traumatismos muitas vezes são advindos de atividades esportivas ${ }^{5,8,13}$.

Ao exame clínico observou-se que apesar dos ferimentos, os lábios e mucosa necessitavam apenas de cuidados simples, com a limpeza dos mesmos. Já o dente apresentou-se fora de posição e radiograficamente observou-se uma fratura horizontal na raiz, em terço médio. Sabe-se que as fraturas dentárias radiculares requerem, além de um preciso diagnóstico, o pronto e correto atendimento para que se possa obter um bom prognóstico do caso, ${ }^{6,13}$.

O diagnóstico dessas fraturas baseia-se na mobilidade clínica do dente, deslocamento do fragmento coronário, na sensibilidade à palpação sobre a raiz e no aspecto radiográfico, sendo importante ainda o diagnóstico pulpar $^{13}$. No caso a decisão baseada na idade do paciente e na condição da fratura foi reposicionar o dente e realizar uma contenção rígida, baseando-se na literatura que preconiza um tratamento inicial de uma fratura radicular consistindo no reposicionamento do fragmento e imobilização do dente lesado aos dentes adjacentes possibilitando a estabilização do mesmo ${ }^{5,14}$.

O reposicionamento foi realizado com auxílio de um fórceps, sob anestesia. $\mathrm{E}$ a contenção rígida foi realizada. $\mathrm{O}$ Splint foi realizado se for utilizado um fio ortodôntico 
fixado com resina composta e posicionado na superfície vestibular dos incisivos.

A decisão por acompanhamento sem a intervenção endodôntica radical veio em consonância com o responsável pela criança e foi observado em longo prazo uma decisão acertada. A base para essa tomada de decisão foi dada por um estudo que indica o reposicionamento dental e a contenção rígida. Todavia a contenção semirrígida também tem mostrado bom prognóstico ${ }^{15}$.

Após um mês o Splint foi removido e o dente manteve a sensibilidade pulpar, sendo assim optou-se por mantê-lo sem intervenção endodôntica. O prognóstico depende de fatores como: grau de mobilidade do fragmento, estágio de desenvolvimento da raiz, localização da fratura e qualidade do tratamento instituído. $\mathrm{O}$ tratamento realizado no caso foi de acordo com a literatura, que mostra a confiabilidade da conduta adotada e sendo assim foi instituído um acompanhamento semanal no primeiro mês, e mensal durante seis meses. E posteriormente a cada seis meses, a fim de confirmar o prognóstico apresentado no início do tratamento e realizar reintervenções se necessárias.

\section{CONCLUSÃO}

Após o controle de dois anos o dente manteve-se vital e sem alteração de cor, mostrando que o tratamento conservador realizado com a manutenção do dente em posição e manutenção da vitalidade pulpar é uma alternativa viável para o tratamento de fraturas radiculares horizontais.

\section{REFERÊNCIAS}

1. Dale RA. Dentoalveolar trauma. Emerg Med Clin North Am. 2000;18(3):521-38.

2. Gassner R, Bösch R, Tuli T, Emshoff R. Prevalence of dental trauma in 6000 patients with facial injuries: implications for prevention. Oral Surg Oral Med Oral Pathol Oral Radiol Endod. 1999;87(1):27-33.

3. Porto RB, Freitas JS, Cruz MR, Bressani AE, Barata JS, Araújo FB. Prevalence of dento-alveolar traumatisms in the urgency pediatric dental clinic of FO-UFRGS. Rev Fac Odontol Porto Alegre. 2003;44:52-6.

4. Andreasen JO, Andreasen FM, Bakland LK, Flores MT. Traumatic dental injuries: a manual. 2nd ed. Oxford: Blackwell; 2003.

5. Sanabe ME, Cavalcante LB, Coldebella CR, Abreu-eLima FCB. Urgências em traumatismos dentários: classificação, características e procedimentos. Rev Paul Pediatr. 2009;27(4):447-51.

6. Oliveira JCM, Silva FSB, Pinto SSL. Fratura radicular horizontal: relato de caso. Rev Bras Odontol. 2008;65(1):76-9.

7. Andreasen JO, Hjörting-Hansen E. Intra-alveolar root fracture: radiographic and histologic study of 50 cases. J Oral Surg. 1967;25:414-26.

8. Andreasen JO, Andreasen FM, Mejàre I, Cvek M. Healing of 400 intra-alveolar root fractures. 1. Effect of pre-injury and injury factors such as sex, age, stage of root development, fracture type, location of fracture and severity of dislocation. Dent Traumatol. 2004;20(4):192-202.

9. May JJ, Cohenca N, Peters OA. Contemporary management of horizontal root fractures to the permanent dentition: diagnosis-radiologic assessment to include cone-beam computed tomography. J Endod. 2013;39(3 Suppl):S20-5.

10. Pan CS, Walker RT. Root fractures: a case of dental non-intervention. Endod Dent Traumatol. 1988;4(4):186-8.

11. Cvek M, Tsilingaridis G, Andreasen JO. Survival of 534 incisors after intra-alveolar root fracture in patients aged 7-17 years. Dent Traumatol. 2008;24(4):379-87.

12. Ozbek M, Serper A, Calt S. Repair of untreated horizontal root fracture: a case report. Dent Traumatol. 2003;19(5):296-7.

13. Zaleckiene V, Peciuliene V, Brukiene V, Drukteinis S. Traumatic dental injuries: etiology, prevalence and possible outcomes. Stomatologija. 2014;16(1):7-14.

14. Zuza EP, Silva AP, Vanzato JW, Toledo BE. Fratura radicular horizontal: relato de um caso com seis anos de proservação. J Bras Clin Estet Odontol 2000;4:21-3.

15. Arikan V, Celikten ZK, Sari S. Treatment of horizontal root fractured central incisors and 30 months follow up: 2 case reports. Eur J Paediatr Dent. 2014;15(2 Suppl):199-202. 